\title{
Biaxial nematic phase in the Maier-Saupe model for a mixture of discs and cylinders
}

\author{
E. F. Henriques and S. R. Salinas \\ Instituto de Física, Universidade de São Paulo \\ Caixa Postal 66318, CEP 05314-970, São Paulo, Brazil
}

14 October 2011

\begin{abstract}
We analyze the global phase diagram of a Maier-Saupe lattice model with the inclusion of disorder degrees of freedom to mimic a mixture of oblate and prolate molecules (discs and cylinders). In the neighborhood of a Landau multicritical point, solutions of the statistical problem can be written as a Landau-de Gennes expansion for the free energy. If the disorder degrees of freedom are quenched, we confirm the existence of a biaxial nematic strucure. If orientational and disorder degrees of freedom are allowed to thermalize, this biaxial solution becomes thermodynamically unstable. Also, we use a two-temperature formalism to mimic the presence of two distinct relaxation times, and show that a slight departure from complete thermalization is enough to stabilize a biaxial nematic phase.
\end{abstract}

\section{Introduction}

The transition between a uniaxial nematic structure and an orientationally disordered phase is perhaps the most investigated and best characterized phase transition phenomenon in liquid crystalline systems [1]. This weak first-order transition is quite well described by the mean-field theory of Maier and Saupe [2][3] [4], which can also be formulated in terms of a fully-connected statistical lattice Hamiltonian [5]. The existence of a biaxial nematic nematic phase, however, and the transitions between different types 
of nematic structures, which have been proposed on the basis of phenomenological calculations for systems with intrinsically biaxial molecular groups [6], turned out to be much more difficult to characterize experimentally [8]. Although there have been some recent reports of a biaxial nematic structure in thermotropic liquid crystalline systems formed by bent-core or boomerangshaped molecules [9], a biaxial phase has been first characterized in the phase diagram of a lyotropic liquid mixture [10] [11], which is better represented by a lattice model of shape-disordered uniaxial molecules. We then revisit the problem of a Maier-Saupe lattice model, with the inclusion of extra degrees of freedom to mimic a mixture of oblate and prolate molecules (discs and cylinders). Slightly different versions of this problem have been analyzed by different authors, with some conflicting results [12, 13, 14, 15]. According to the conclusions of a recent calculation for a shape-disordered Maier-Saupe model with restricted orientations [16], we point out that the introduction of two sets of degrees of freedom opens the possibility of choosing different relaxation times, with different outcomes for the thermodynamic stability of a biaxial nematic structure.

We then formulate and analyze a Maier-Saupe lattice model for a mixture of discs and cylinders. This problem includes orientational (quadrupolar) and shape-disordered (discs and cylinders) degrees of freedom, which might be associated with different relaxation times. Solutions can be obtained by the application of well-known methods of statistical mechanics. First, we treat the case of fixed (frozen) disorder, as in a typical problem of a disordered solid state system. In this quenched case, disorder (shape) degrees of freedom are fixed, frozen, while the orientational degrees of freedom are allowed to thermalize during experimental times. We then treat the case of annealed (thermalized) disorder, in which both orientational and disorder degrees of freedom are allowed to reach thermodynamic equilibrium during experimental times, and which is certainly more adequate to explain the behavior of a liquid system. In agreement with previous calculations for similar models, in the quenched case we show the existence of a biaxial nematic structure for typical distributions of shape-disorder [13. In the thermalized case, however, there is a biaxial nematic solution of the model equations, but it turns out to be thermodynamically unstable (in agreement with older calculations by Palffy-Murhoray and collaborators [12]). We then resort to a formalism based on two heat reservoirs, at distinct temperatures, which is conceived to describe an intermediate situation, between fully fixed and fully thermalized distributions of disorder variables [17]. As in the calculations for the 
Maier-Saupe model with restricted orientations [16], we show that a small difference of temperatures, which is equivalent to a slight departure from complete thermalization, is already sufficient to produce a stable biaxial nematic phase.

It should be pointed out that we write closed-form solutions of the statistical problem, which are not restricted to the neighborhood of the transitions, and which can be used to draw global phase diagrams, in terms of temperature and either concentration or chemical potential. Also, it is feasible to extend these calculations beyond the mean-field level, as it has been done in the annealed case for the analogous model with restricted orientations [18]. We use the model solutions to write a Landau-de Gennes expansion for the free energy in terms of the invariants of the tensor order parameter, but with model-dependent coefficients. Corresponding phenomenological expansions have been investigated by a number of authors [2] [19][20], and we can use some asymptotic results to confirm the numerical analysis in the neighborhood of a Landau multicritical point.

\section{Disordered Maier-Saupe model}

The Maier-Saupe theory of the nematic-isotropic phase transition can be obtained from a statistical calculation for a fully-connected lattice model given by the quadrupolar Hamiltonian

$$
\mathcal{H}\left\{\vec{n}_{i}\right\}=-\sum_{1 \leq i<j \leq N} \frac{A}{N} \sum_{\mu, \nu=x, y, z} S_{i}^{\mu \nu} S_{j}^{\mu \nu},
$$

where the sum is over all pairs of lattice sites, $A / N$ is a scaled interaction energy, and $\left\{S_{i}^{\mu \nu}\right\}$ is a set orientational (quadrupolar) variables, given by

$$
S_{i}^{\mu \nu}=\frac{1}{2}\left(3 n_{i}^{\mu} n_{i}^{\nu}-\delta_{\mu \nu}\right)
$$

where $\overrightarrow{n_{i}}=\left(n_{i}^{x}, n_{i}^{y}, n_{i}^{z}\right)$, with $\left|\vec{n}_{i}\right|=1$, is a local nematic director. This meanfield Maier-Saupe (MS) model is known to reproduce the main features of the (weak) first-order transition between uniaxial nematic and disordered phases.

We mimic the behavior of a binary mixture of oblate and prolate molecules (discs and cylinders) by introducing an additional set of (shape) degrees of 
freedom, $\left\{\lambda_{i}\right\}$, with $\lambda_{i}= \pm 1$, for $i=1, \ldots, N$. Given the configurations of orientational and disorder (shape) degrees of freedom, $\left\{\vec{n}_{i}\right\}$ and $\left\{\lambda_{i}\right\}$, the simplest Maier-Saupe Hamiltonian for this mixture of discs and cylinders is given by

$$
\mathcal{H}\left(\left\{\lambda_{i}\right\},\left\{\vec{n}_{i}\right\}\right)=-\sum_{1 \leq i<j \leq N} \frac{A}{N} \lambda_{i} \lambda_{j} \sum_{\mu, \nu=x, y, z} S_{i}^{\mu \nu} S_{j}^{\mu \nu}
$$

which can also be written in the more convenient form

$$
\mathcal{H}\left(\left\{\lambda_{i}\right\},\left\{\vec{n}_{i}\right\}\right)=-\frac{A}{2 N} \sum_{\mu, \nu=x, y, z}\left[\sum_{i=1}^{N} \lambda_{i} S_{i}^{\mu \nu}\right]^{2}
$$

where we have discarded irrelevant terms in the $N \rightarrow \infty$ limit.

In a typical problem of a disordered system of solid state physics, the disordered degrees of freedom are fixed, frozen, while the orientational degrees of freedom are allowed to thermalize during the experimental times [21]. In this fixed, quenched case, as in amorphous and glassy materials, disorder variables are not strictly thermodynamic. In the opposite case, which seems more adequate to describe liquid mixtures, both orientational and disorder degrees of freedom are allowed to reach thermodynamic equilibrium during experimental times. The fully thermalized, annealed case, is then treated according to the standard rules of equilibrium thermodynamics.

In the following paragraphs, we consider quenched and annealed cases separately. As in the work of Henriques and Henriques [13], for a lattice Maier-Saupe model with restricted orientations, we confirm that there is a biaxial nematic structure in the quenched case. Also, we show that this biaxial structure becomes thermodynamically unstable in the annealed case, which agrees with an older Maier-Saupe calculation for a mixture of cylinders and discs by Palffy-Muhoray and collaborators [12]. We then introduce the two-temperature formalism [16] [17] in order to show that a slight departure from complete thermalization is already sufficient to produce a stable biaxial nematic phase. The numerical analysis of the free energy is supplemented, and confirmed, by an analysis of a Landau-de Gennes expansion in the neighborhood of the Landau multicritical point. 


\subsection{Quenched disorder}

Given the set of disorder variables, $\left\{\lambda_{i}\right\}$, we write the canonical partition function

$$
Z\left(\left\{\lambda_{i}\right\}\right)=\sum_{\left\{\overrightarrow{n_{i}}\right\}} \exp \left\{\frac{\beta}{2 N} \sum_{\mu, \nu}\left[\sum_{i=1}^{N} \lambda_{i} S_{i}^{\mu \nu}\right]^{2}\right\}
$$

where $\beta=A / k_{B} T=1 / t$ is the inverse of a (dimensionless) temperature, $\mu, \nu=1,2,3$ correspond to the Cartesian directions, and we are summing over configurations of the local (microscopic) directors. In this quenched case, $\left\{\lambda_{i}\right\}$ is a set of independent, identical, and identically distributed random variables, associated with a probability distribution

$$
P\left(\left\{\lambda_{i}\right\}\right)=\prod_{i=1}^{N} p\left(\lambda_{i}\right)
$$

It is convenient to parametrize the local directors by polar coordinates,

$$
\overrightarrow{n_{i}}=\left(\sin \theta_{i} \cos \phi_{i}, \sin \theta_{i} \sin \phi_{i}, \cos \theta_{i}\right),
$$

with $\Omega_{i}=\left(\theta_{i}, \phi_{i}\right)$, so that

$$
S_{i}^{\mu \nu}=\frac{3}{2}\left(\begin{array}{ccc}
\sin ^{2} \theta_{i} \cos ^{2} \phi_{i}-\frac{1}{3} & \sin ^{2} \theta_{i} \sin \phi_{i} \cos \phi_{i} & \sin \theta_{i} \cos \theta_{i} \cos \phi_{i} \\
\sin ^{2} \theta_{i} \sin \phi_{i} \cos \phi_{i} & \sin ^{2} \theta_{i} \sin ^{2} \phi_{i}-\frac{1}{3} & \sin \theta_{i} \cos \theta_{i} \sin \phi_{i} \\
\sin \theta_{i} \cos \theta_{i} \cos \phi_{i} & \sin \theta_{i} \cos \theta_{i} \sin \phi_{i} & \cos ^{2} \theta_{i}-\frac{1}{3}
\end{array}\right),
$$

and the sum over orientational configurations becomes an integral over solid angles,

$$
Z\left(\left\{\lambda_{i}\right\}\right)=\prod_{i} \int d \Omega_{i} \exp \left\{\frac{\beta}{2 N} \sum_{\mu, \nu=x, y, z}\left[\sum_{i=1}^{N} \lambda_{i} S^{\mu \nu}\left(\Omega_{i}\right)\right]^{2}\right\} .
$$

In the thermodynamic limit $(N \rightarrow \infty)$, we have the asymptotic form

$$
Z\left(\left\{\lambda_{i}\right\}\right) \sim \exp \left[-\beta N g_{N}\left(\left\{\lambda_{i}\right\}\right)\right] .
$$

The resulting (quenched) free energy $g_{q}$ comes from an average of $g_{N}\left(\left\{\lambda_{i}\right\}\right)$ over the distribution of shape variables $P\left(\left\{\lambda_{i}\right\}\right)$,

$g_{q} \sim\left\langle g\left(\left\{\lambda_{i}\right\}\right)\right\rangle \sim \frac{1}{N}\left\langle\ln Z\left(\left\{\lambda_{i}\right\}\right)\right\rangle=\frac{1}{N} \int\left(\prod_{i} d \lambda_{i}\right) P\left(\left\{\lambda_{i}\right\}\right) \ln Z\left(\left\{\lambda_{i}\right\}, \beta\right)$, 
where the brackets $\langle\ldots\rangle$ indicate disorder averages, and we are taking the limit of large $N$.

The sum over the square terms in equation (9), can be dealt with by a set of Gaussian identities. For example, we have

$$
\begin{gathered}
\exp \left\{\frac{\beta}{2 N}\left[\sum_{i} \lambda_{i} S^{11}\left(\Omega_{i}\right)\right]^{2}\right\}= \\
=\int_{-\infty}^{+\infty} \frac{d x_{11}}{\sqrt{\pi}} \exp \left\{-x_{11}^{2}+2\left(\frac{\beta}{2 N}\right)^{1 / 2}\left[\sum_{i=1}^{N} \lambda_{i} S^{11}\left(\Omega_{i}\right)\right] x_{11}\right\}= \\
=\left(\frac{\beta N}{2 \pi}\right)^{1 / 2} \int_{-\infty}^{+\infty} d q_{11} \exp \left\{-\frac{1}{2} N \beta q_{11}^{2}+\sum_{i=1}^{N} \beta \lambda_{i} S^{11}\left(\Omega_{i}\right) q_{11}\right\} .
\end{gathered}
$$

Taking into account the symmetry of the traceless tensor $S^{\mu \nu}$, we introduce a set of six variables, $q_{11}, q_{22}, q_{33}, q_{12}, q_{13}$, and $q_{23}$, and write the partition function

$$
Z\left(\left\{\lambda_{i}\right\}\right)=\int[d q] \exp \left\{-\frac{1}{2} N \beta \sum_{\mu} q_{\mu \mu}^{2}-N \beta \sum_{\mu<\nu} q_{\mu \nu}^{2}+\sum_{i=1}^{N} \ln M_{i}\right\},
$$

where

$$
[d q]=\left(\frac{\beta N}{2 \pi}\right)^{3} d q_{11} d q_{22} d q_{33} d q_{12} d q_{13} d q_{23}
$$

and

$$
M_{i}=M\left(\lambda_{i},\left\{q_{\mu \nu}\right\}\right)=\int d \Omega_{i} \exp \left[\sum_{\mu \leq \nu} \beta \lambda_{i} S^{\mu \nu}\left(\Omega_{i}\right) q_{\mu \nu}\right] .
$$

In the thermodynamic limit, we resort to Laplace's asymptotic method, and invoke the law of large numbers,

$$
\frac{1}{N} \sum_{i=1}^{N} \ln M\left(\lambda_{i},\left\{q_{\mu \nu}\right\}\right) \longrightarrow\left\langle\ln M\left(\lambda_{i},\left\{q_{\mu \nu}\right\}\right)\right\rangle=\int d \lambda p(\lambda) \ln M\left(\lambda,\left\{q_{\mu \nu}\right\}\right)
$$

We then have a self-averaged expression for the quenched free energy,

$$
g_{q}=\frac{1}{2}\left(q_{11}^{2}+q_{22}^{2}+q_{33}^{2}\right)+q_{12}+q_{13}+q_{23}-\frac{1}{\beta} \int d \lambda p(\lambda) \ln M\left(\lambda,\left\{q_{\mu \nu}\right\}\right),
$$


where the set of parameters $\left\{q_{\mu \nu}\right\}$ come from the minima of the asymptotic integration,

$$
q_{\delta \delta}=\int \lambda p(\lambda) d \lambda \frac{\int d \Omega S^{\delta \delta}(\Omega) \exp \left[\sum_{\mu \leq \nu} \beta \lambda S^{\mu \nu}(\Omega) q_{\mu \nu}\right]}{\int d \Omega \exp \left[\sum_{\mu \leq \nu} \beta \lambda S^{\mu \nu}(\Omega) q_{\mu \nu}\right]},
$$

for $\delta=1,2,3$, and

$$
q_{\delta \gamma}=\frac{1}{2} \int \lambda p(\lambda) d \lambda \frac{\int d \Omega S^{\delta \gamma}(\Omega) \exp \left[\sum_{\mu \leq \nu} \beta \lambda S^{\mu \nu}(\Omega) q_{\mu \nu}\right]}{\int d \Omega \exp \left[\sum_{\mu \leq \nu} \beta \lambda S^{\mu \nu}(\Omega) q_{\mu \nu}\right]},
$$

for $\delta<\gamma$. The set of variables $\left\{q_{\mu \nu}\right\}$ has a clear physical interpretation as the mean values of the quadrupole tensor components. In fact, if we include field terms in the original Hamiltonian, of the form $h^{\mu \nu} \lambda_{i} S_{i}^{\mu \nu}$, with couplings of local quadrupoles $\lambda_{i} S_{i}^{\mu \nu}$ to external fields $h^{\mu \nu}$, the (Gibbs) free energy will depend on these external fields, and the mean quadrupoles will be given by $q_{\mu \nu}=-\partial g_{q} / \partial h^{\mu \mu}$.

Using the explicit forms of $S^{\mu \nu}(\Omega)$, given by equation (8), it is straightforward to show that we can choose $q_{12}=q_{13}=q_{23}=0$, with $q_{11}, q_{22}, q_{33} \neq 0$. This self-consistent choice leads to a diagonal mean-quadrupole tensor in a convenient laboratory frame of reference. We then write the quenched free energy

$g_{q}=\frac{1}{2}\left(q_{11}^{2}+q_{22}^{2}+q_{33}^{2}\right)-\frac{1}{\beta} \int p(\lambda) d \lambda \ln \left\{\int d \Omega \exp \left[\beta \lambda \sum_{\mu=1,2,3} S^{\mu \mu}(\Omega) q_{\mu \mu}\right]\right\}$,

where

$$
q_{\mu \mu}=\int \lambda p(\lambda) d \lambda \frac{\int d \Omega S^{\mu \mu}(\Omega) \exp \left[\sum_{\mu} \beta \lambda S^{\mu \mu}(\Omega) q_{\mu \mu}\right]}{\int d \Omega \exp \left[\sum_{\mu} \beta \lambda S^{\mu \mu}(\Omega) q_{\mu \mu}\right]} .
$$


Also, we remark that

$$
\sum_{\mu} q_{\mu \mu}=0
$$

which confirms the traceless property of the mean-quadrupole tensor. Therefore, we introduce the standard parametrization

$$
\mathbf{q}=\left(\begin{array}{ccc}
q_{11} & 0 & 0 \\
0 & q_{22} & 0 \\
0 & 0 & q_{33}
\end{array}\right)=\frac{1}{2}\left(\begin{array}{ccc}
\eta-s & 0 & 0 \\
0 & -\eta-s & 0 \\
0 & 0 & 2 s
\end{array}\right)
$$

so that (i) $s \neq 0$ and $\eta \neq 0$ in a biaxial nematic phase, (ii) $s \neq 0$ and $\eta=0$ in a uniaxial nematic phase, and (iii) $s=0$ and $\eta=0$ in the disordered phase.

The analysis of the quenched free energy depends on the choice of the distribution $p(\lambda)$. For example, we may choose

$$
p(\lambda)=c \delta(\lambda-1)+(1-c) \delta(\lambda+1),
$$

which represents a sample with a number concentration $c$ of prolate molecules $(\lambda=+1)$ and $1-c$ of oblate molecules $(\lambda=-1)$, and which is convenient for comparisons with the annealed situation. If we adopt this form of $p(\lambda)$, it is straightforward to analyze equations (20) and (21), with the standard parametrization (23), and draw the phase diagram of figure (1). We indicate two uniaxial nematic phases, $N_{+}$with $s>0$, and $N_{-}$with $s<0$, separated by a first-order boundary (heavy dashed line) from the isotropic phase. The biaxial nematic region is limited by two critical lines that meet at the Landau multicritical point $\left(c_{L}=1 / 2\right.$ and $\left.t_{L}=1 / \beta_{L}=3 / 10\right)$. In the neighborhood of this Landau point, we confirm these results by the analysis of an expansion

of the free energy in terms of the invariants of the tensor order parameter.

\section{$2.2 \quad$ Annealed disorder}

In the annealed approach, we write the canonical partition function

$$
Z=\sum_{\left\{\lambda_{i}\right\}}^{\prime} \sum_{\left\{\vec{n}_{i}\right\}} \exp \left\{\frac{\beta}{2 N} \sum_{\mu, \nu}\left[\sum_{i=1}^{N} \lambda_{i} S_{i}^{\mu \nu}\right]^{2}\right\},
$$

where the sum over the shape configurations $\left\{\lambda_{i}\right\}$ is restricted by the fixed value of the number density $c$ of prolate molecules,

$$
\sum_{i=1}^{N} \lambda_{i}=N_{+}-N_{-}=N(2 c-1)
$$




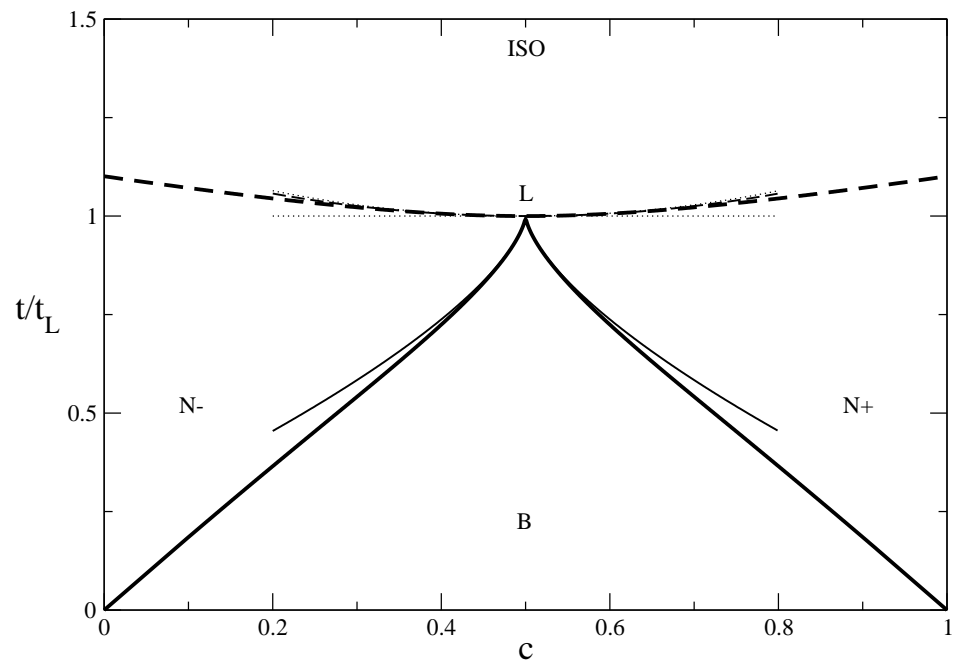

Figure 1: Phase diagram, in terms of the ration between temperature $t / t_{L}$ and the convcentration $c$, for the case of quenched disorder with a doubledelta distribution. We indicate the nematic biaxial $(B)$, two uniaxial nematic phases of opposite symmetry $\left(N_{+}\right.$and $\left.N_{-}\right)$and the Landau multicritical point $(L)$, located at $t_{L}=3 / 10$ and $c_{L}=0.5$. The heavy dashed line corresponds to first-order transitions. Heavy solid lines correspond to continuous transitions (between biaxial an uniaxial nematic structures). We also indicate asymptotic results coming from an expansion of the free energy in the neighborhood ot the Landau multicritical point (thin solid lines). The stability curves for the isotropic and nematic phases are given by the dotted lines. 
It is then convenient to introduce a chemical potential $\mu$ and to change to a grand canonical ensemble,

$$
\Xi=\sum_{\left\{\lambda_{i}\right\}} \sum_{\left\{\vec{n}_{i}\right\}} \exp \left\{\frac{1}{2} \beta \mu\left[N+\sum_{i=1}^{N} \lambda_{i}\right]+\frac{\beta}{2 N} \sum_{\mu, \nu}\left[\sum_{i=1}^{N} \lambda_{i} S_{i}^{\mu \nu}\right]^{2}\right\} .
$$

In analogy with the treatment of the quenched case, we use a polar parametrization for $S^{\mu \nu}$, take advantage of the Gaussian identities to eliminate the squares, and write the asymptotic $(N \rightarrow \infty)$ result

$$
\Xi \sim \exp [-\beta N \phi]
$$

where $\phi$ is a grand potential per molecule,

$$
\phi=-\frac{\mu}{2}+\frac{1}{2}\left(q_{11}^{2}+q_{22}^{2}+q_{33}^{2}\right)-\frac{1}{\beta} \ln \zeta
$$

with

$$
\begin{aligned}
& \zeta=\left[\exp \left(\frac{1}{2} \beta \mu\right)\right] \int d \Omega \exp \left[\beta L\left(\Omega,\left\{q_{\mu \mu}\right\}\right)\right]+ \\
& +\left[\exp \left(-\frac{1}{2} \beta \mu\right)\right] \int d \Omega \exp \left[-\beta L\left(\Omega,\left\{q_{\mu \mu}\right\}\right)\right]
\end{aligned}
$$

and

$$
L\left(\Omega,\left\{q_{\mu \mu}\right\}\right)=S^{11}(\Omega) q_{11}+S^{22}(\Omega) q_{22}+S^{33}(\Omega) q_{33},
$$

which should be supplemented by the coupled equations for the minimization of $\phi$ with respect to $q_{11}, q_{22}$ and $q_{33}$. Again, we see that the mean quadrupole tensor is traceless, so that we can use the standard parametrization of equation (23).

The analysis of the free energy shows that the biaxial solution $(s \neq 0$, $\eta \neq 0$ ) is thermodynamically unstable (it is a kind of saddle-point instead of a minimum of $\phi$ ). We then draw the phase diagram of figure (2), in terms of the thermodynamic field variables $t=1 / \beta$, dimensionless temperature, and chemical potential $\mu$. The dashed lines indicate first-order boundaries between the uniaxial nematic phases $N_{+}$and $N_{-}$, and between the isotropic and each one of the nematic phases. The multicritical Landau point (at $\mu=0$ and $\left.t_{L}=3 / 10\right)$ is just a simple triple point. We can also draw the phase diagram shown in figure (3), in terms of temperature and concentration, which may be more interesting from the experimental point of view. 


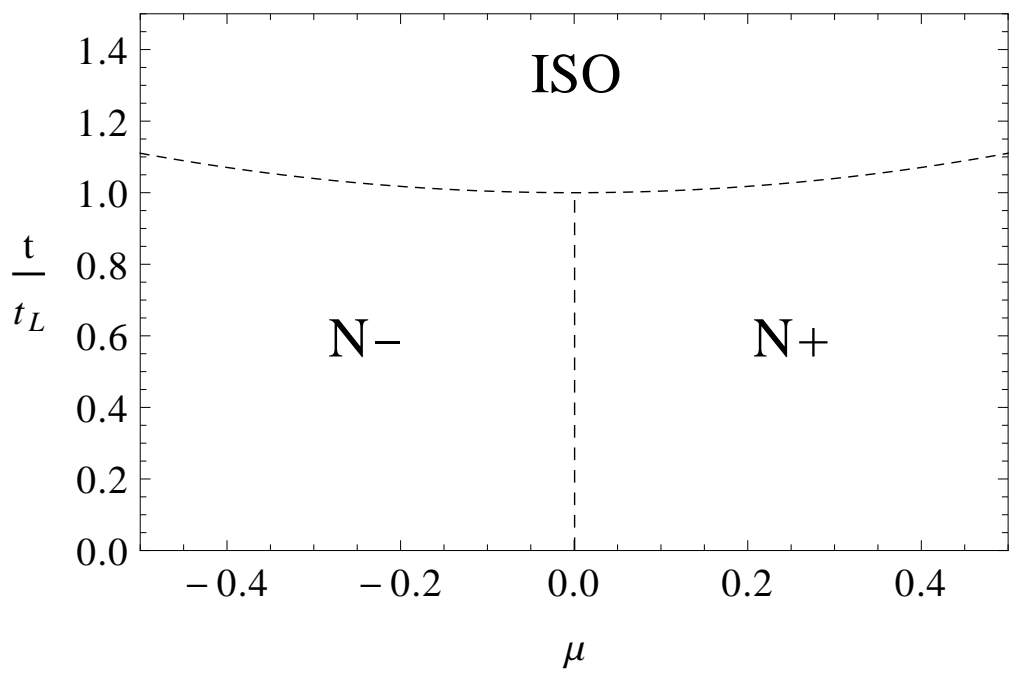

Figure 2: Phase diagram for the annealed case in terms of thermodynamic field variables (dimensionless temperature $t=1 / \beta$ and chemical potential $\mu$ ). The triple point is located at $t_{L}=3 / 10$ and $\mu=0$. Dashed lines indicate first-order boundaries.

The tie lines in the ordered region indicate the coexistence of two distinct uniaxial nematic phases. Again, we confirm these results by an analysis of an expansion of the free energy in terms of the invariants of the tensor order parameter.

\subsection{Two-temperature formalism}

In the two-temperature formalism, we introduce two heat baths, at different temperatures, associated with the relaxation times of the orientational (quicker) and disorder (slower) degrees of freedom. We now give a brief account of this formalism [17]. Given a configuration $\left\{\lambda_{i}\right\}$ of the slower disorder variables, we can schematically write the probability of occurrence of a configuration $\left\{\sigma_{i}\right\}$ of the orientational variables,

$$
P\left(\left\{\sigma_{i}\right\} \mid\left\{\lambda_{i}\right\}\right)=\frac{1}{Z_{\sigma}} \exp \left[-\beta \mathcal{H}\left(\left\{\sigma_{i}\right\},\left\{\lambda_{i}\right\}\right)\right],
$$




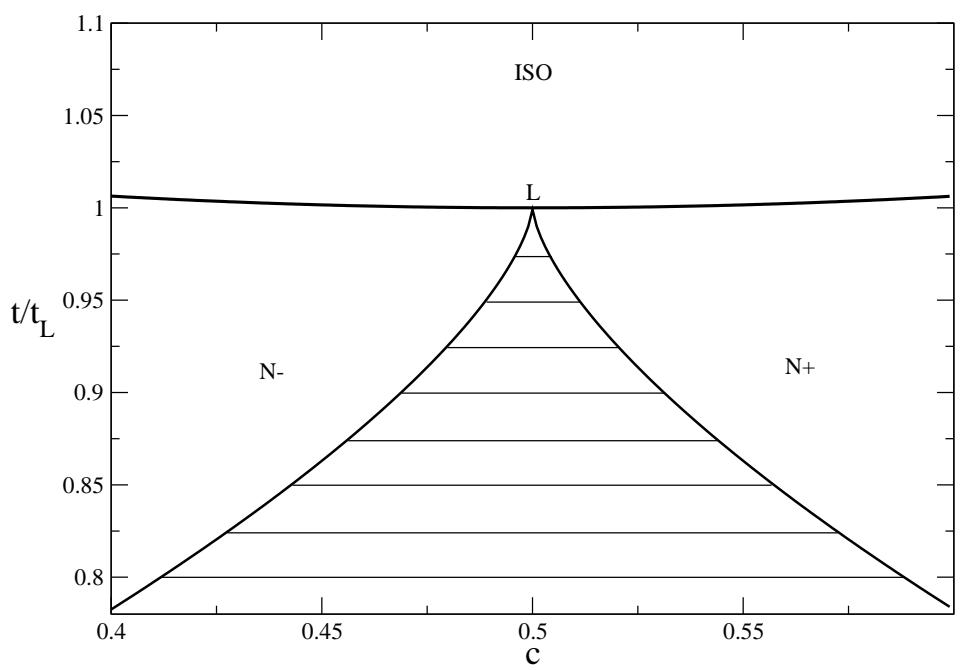

Figure 3: Phase diagram, in terms of temperature and concentration, in the annealed case. The tie lines indicate the coexistence of two uniaxial nematic phases. The region of coexistence of the uniaxial nematic and the isotropic phases is too narrow to be represented in this graph.

where $T=1 / \beta$ is the temperature of a heat bath, and

$$
Z_{\sigma}=Z_{\sigma}\left(\left\{\lambda_{i}\right\}\right)=\sum_{\left\{\sigma_{i}\right\}} \exp \left[-\beta \mathcal{H}\left(\left\{\sigma_{i}\right\},\left\{\lambda_{i}\right\}\right)\right]
$$

The time evolution of $\lambda_{i}$ is given by a Langevin equation,

$$
\Gamma \frac{\partial \lambda_{i}}{\partial t}=-z(t) \lambda_{i}-\frac{\partial \mathcal{H}}{\partial \lambda_{i}}+\eta_{i}(t)
$$

where $z(t)$ is a multiplier associated with the chemical potential, and

$$
\left\langle\eta_{i}(t) \eta_{j}\left(t^{\prime}\right)\right\rangle=2 \Gamma T_{\lambda} \delta_{i j} \delta\left(t^{\prime}-t^{\prime}\right),
$$

where we have introduced the temperature $T_{\lambda}$ of a second heat bath. With the assumption of quick and slow time scales, it is reasonable to replace $\partial \mathcal{H} / \partial \lambda_{i}$ by its average value,

$$
\frac{\partial \mathcal{H}}{\partial \lambda_{i}} \Longrightarrow\left\langle\frac{\partial \mathcal{H}}{\partial \lambda_{i}}\right\rangle_{\sigma}=\frac{\partial \mathcal{H}_{e f f}}{\partial \lambda_{i}}
$$


where

$$
\mathcal{H}_{e f f}=\mathcal{H}_{e f f}\left(\left\{\lambda_{i}\right\}\right)=-k_{B} T \ln \sum_{\left\{\sigma_{i}\right\}} \exp \left[-\beta \mathcal{H}\left(\left\{\sigma_{i}\right\},\left\{\lambda_{i}\right\}\right)\right]
$$

We then assume that the probability of a configuration $\left\{\lambda_{i}\right\}$ is given by the grand-canonical expression

$$
P(\lambda)=\frac{1}{\Xi\left(\beta_{\lambda}, \beta, N, \mu\right)} \exp \left[\beta_{\lambda} \mu N_{p}-\beta_{\lambda} \mathcal{H}_{e f f}\right]
$$

where

$\Xi\left(\beta_{\lambda}, \beta, N, \mu\right)=\int[d \lambda]\left\{\sum_{\{\sigma\}} \exp \left[-\beta \mathcal{H}\left(\left\{\sigma_{i}\right\},\left\{\lambda_{i}\right\}\right)+\frac{\beta \mu}{2}\left(\sum_{i} \lambda_{i}+N\right)\right]\right\}^{\mathbf{n}}$,

and the ratio $n=T / T_{\lambda}$ resembles the number of replicas in spin-glass problems [21].

According to this two-temperature formalism, the orientational degrees of freedom of the Maier-Saupe model are allowed to reach thermal equilibrium at a temperature $T$ and the disorder degrees of freedom thermalize at a temperature $T_{\lambda} \neq T$, with $n=T / T_{\lambda}$. The problem is then reduced to the calculation of the grand-canonical partition function of $n$ replicas of the original system [16],

$\Xi_{t w o}=\sum_{\left\{\lambda_{i, \alpha}\right\}} \sum_{\left\{\vec{n}_{i, \alpha}\right\}} \exp \left\{\sum_{\alpha=1}^{n}\left[\frac{\beta \mu}{2}\left(N+\sum_{i=1}^{N} \lambda_{i, \alpha}\right)+\frac{\beta}{2 N} \sum_{\mu, \nu}\left(\sum_{i=1}^{N} \lambda_{i, \alpha} S_{i, \alpha}^{\mu \nu}\right)^{2}\right]\right\}$.

In the thermodynamic limit, we write

$$
\Xi_{t w o} \sim \exp \left[-\beta N \phi_{t w o}\right]
$$

where

$$
\phi_{t w o}=-\frac{1}{2} \mu n+\frac{1}{2} n\left(q_{11}^{2}+q_{22}^{2}+q_{33}^{2}\right)-\frac{1}{\beta} \ln \zeta_{t w o}
$$

with

$$
\zeta_{\text {two }}=\left\{\left[\exp \left(\frac{1}{2} \beta \mu\right)\right] \int d \Omega \exp \left[\beta L\left(\Omega,\left\{q_{\mu \mu}\right\}\right)\right]\right\}^{n}+
$$




$$
+\left\{\left[\exp \left(-\frac{1}{2} \beta \mu\right)\right] \int d \Omega \exp \left[-\beta L\left(\Omega,\left\{q_{\mu \mu}\right\}\right)\right]\right\}^{n} .
$$

The minimization of the grand potential $\phi_{t w o}$ leads to the equilibrium values $q_{11}, q_{22}$, and $q_{33}=-q_{11}-q_{22}$. Note that we regain the annealed case for $n=1$, and that the role of this parameter $n$ will become clear in the next Section.

\section{Connections with the Landau-de Gennes expansion}

We have already chosen a standard order parameter, given by the traceless diagonal tensor (23). We then introduce the second and third-order invariants,

$$
I_{2}=\operatorname{Tr}\left[\mathbf{q}^{2}\right]=q_{11}^{2}+q_{22}^{2}+q_{33}^{2}=\frac{1}{2}\left(3 s^{2}+\eta^{2}\right)
$$

and

$$
I_{3}=\operatorname{Tr}\left[\mathbf{q}^{3}\right]=q_{11}^{3}+q_{22}^{3}+q_{33}^{3}=\frac{3}{4} s\left(s^{2}-\eta^{2}\right),
$$

in terms of which it is usual to write the phenomenological Landau-de Gennes expansion for the free energy in the neighborhood of a transition,

$$
f=f_{0}+\frac{A}{2} I_{2}+\frac{B}{3} I_{3}+\frac{C}{4}\left(I_{2}\right)^{2}+\frac{D}{5} I_{2} I_{3}+\frac{E}{6}\left(I_{3}\right)^{2}+\frac{E^{\prime}}{6}\left(I_{2}\right)^{3}+\ldots
$$

The Landau multicritical point is given by $A=B=0$. The stability conditions of the ordered phases in the neighborhood of the Landau point are discussed in terms of the signs of the coefficients of the higher-order terms. It has been shown that $E>0$ is a necessary condition for the stability of a biaxial nematic phase in the vicinity of the Landau multicritical point [2] [19].

In the present case, we remark that it is more convenient to adopt an alternative parametrization, in terms of two new variables $r$ and $\psi$, such that

$$
\begin{aligned}
& q_{11}=-\frac{r}{2}(\cos \psi+\sqrt{3} \sin \psi), \\
& q_{22}=-\frac{r}{2}(\cos \psi-\sqrt{3} \sin \psi),
\end{aligned}
$$

and

$$
q_{33}=r \cos \psi .
$$


We then have $q_{11}+q_{22}+q_{33}=0$,

$$
I_{2}=\frac{3}{2} r^{2}
$$

and

$$
I_{3}=\frac{3}{4} r^{3} \cos (3 \psi)
$$

\subsection{Quenched disorder}

In the neighborhood of the Landau multicritical point, the quenched free energy, given by equation (20), leads to the expansion

$$
\begin{gathered}
g_{q}=g_{0}+\frac{1}{2}\left(1-\frac{3 \beta \overline{\lambda^{2}}}{10}\right) I_{2}-\frac{10 \overline{\lambda^{3}}}{21\left(\overline{\lambda^{2}}\right)^{2}} I_{3}+ \\
+\frac{5 \overline{\lambda^{4}}}{42\left(\overline{\lambda^{2}}\right)^{3}} I_{2}^{2}+\frac{100 \overline{\lambda^{5}}}{231\left(\overline{\lambda^{2}}\right)^{2}} I_{2} I_{3}+\frac{2000 \overline{\lambda^{6}}}{7007\left(\overline{\lambda^{2}}\right)^{5}} I_{3}^{2}-\frac{1450 \overline{\lambda^{6}}}{27027\left(\overline{\lambda^{2}}\right)^{5}} I_{2}^{3}
\end{gathered}
$$

where $g_{0}$ is the free energy of the isotropic phase,

$$
\overline{\lambda^{k}}=\int \lambda^{k} p(\lambda) d \lambda
$$

and we have kept terms up to fifth order only. The Landau multicritical point is given by $t=3 \overline{\lambda^{2}} / 10$ and $\overline{\lambda^{3}}=0$ (for an arbitrary distribution of shapes).

Let us choose the double-delta distribution, given by equation (24), which is particularly adequate for a comparison with the annealed case. The expansion of the free energy in the neighborhood of the Landau multicritical point $\left(\beta=\beta_{L}=10 / 3\right.$ and $\left.c=c_{L}=1 / 2\right)$ is given by

$$
\begin{gathered}
g_{q}=-\frac{\ln (4 \pi)}{\beta}+\frac{1}{2}\left(1-\frac{3 \beta}{10}\right) I_{2}-\frac{10}{21}(2 c-1) I_{3}+ \\
+\frac{5}{42} I_{2}^{2}+\frac{2000}{7007} I_{3}^{2}-\frac{1450}{27027} I_{2}^{3} .
\end{gathered}
$$

The positive sign of the coefficient of $I_{3}^{2}$ indicates that biaxial nematic phase is stable in the neighborhood of this Landau point. Sufficiently close to 
the Landau point, we can show that the first-order transition between the uniaxial nematic and the disordered phase is asymptotically given by

$$
\frac{t}{t_{L}}=1+\frac{10}{63}(2 c-1)^{2}
$$

in agreement with numerical calculations (see figure 1). Also, we show that the critical lines separating the biaxial and the two uniaxial nematic phases are given by

$$
\frac{t}{t_{L}}=1-\frac{10}{21}(6)^{1 / 3}\left(\frac{1001}{1200}\right)^{2 / 3}(2 c-1)^{2 / 3},
$$

which also agrees with numerical calculations close to the Landau point (see figure 1). This phase diagram, with a stable biaxial nematic phase, is in qualitative agreement with previous results for a Maier-Saupe model with restricted orientations [13] 16]. Using the notation of the phenomenological Landau-de Gennes free energy, we remark that $C>0$ and $D=0$, as in the work of Allender and Longa [19]. However, new topologies may arise if we consider other forms of the distribution $p(\lambda)$.

\subsection{Annealed disorder}

In the annealed case, we use the grand potential $\phi$, given by equation (26), to locate the Landau multicritical point $\left(\beta_{L}=10 / 3\right.$ and $\left.\mu_{L}=0\right)$, and write the expansion

$$
\begin{aligned}
\phi & =\phi_{0}+\frac{1}{2}\left(1-\frac{3 \beta}{10}\right) I_{2}-\frac{50}{63} \mu I_{3}+ \\
& +\frac{5}{42} I_{2}^{2}-\frac{2500}{27027} I_{3}^{2}-\frac{1450}{27027} I_{2}^{3}
\end{aligned}
$$

where

$$
\phi_{0}=-\frac{1}{2} \mu-\frac{1}{\beta} \ln \left[8 \pi \cosh \left(\frac{1}{2} \beta \mu\right)\right]
$$

is the grand potential of the isotropic phase. The negative coefficient of $I_{3}^{2}$ shows that there is no stable biaxial nematic phase in the neighborhood of the Landau multicritical point. The line at $\mu=0$, below the temperature of the Landau point, is a first-order boundary between two distinct uniaxial nematic phase (with $s>0$ and $s>0$ ). We show that the first-order lines 
separating the uniaxial nematic from the isotropic phase are given by the asymptotic expression

$$
\frac{t}{t_{L}}=1+\frac{250}{567} \mu^{2}
$$

in the immediate vicinity of the Landau point. The phase diagram in figure 2 is in agreement with these asymptotic results.

We can also calculate some asymptotic expressions in terms of temperature and concentration, which are more convenient variables from the experimental point of view. For example, the region of coexistence of the uniaxial nematic phases in figure 3 is limited by the asymptotic border

$$
\frac{t}{t_{L}}=1-\frac{5}{7}\left(\frac{42}{25}\right)^{2 / 3}\left(c-\frac{1}{2}\right)^{2 / 3} .
$$

The asymptotic form of the first-order border between the uniaxial nematic and the isotropic phases is given by

$$
\frac{t}{t_{L}}=1+\frac{40}{63}\left(c-\frac{1}{2}\right)^{2}
$$

in full agreement with numerical calculations. Also, it should be remarked that the phase diagram in figure 3 is in qualitative agreement with previous calculations for a Maier-Saupe model with restricted orientations [16].

\subsection{Two-temperature formalism}

The same sort of calculations can be carried out for the grand potential in the two-temperature formalism. From equation (38), in the immediate neighborhood of the Landau point, we have the expansion

$$
\begin{gathered}
\phi_{t w o}=\phi_{0, t w o}+\frac{1}{2}\left(1-\frac{3 \beta}{10}\right) n I_{2}-\frac{50}{63} \frac{\mu}{n} I_{3}+ \\
\frac{5}{42} \frac{1}{n^{2}} I_{2}^{2}+\frac{2500}{27027} \frac{1}{n^{4}}\left(\frac{108}{35}-\frac{143 n}{35}\right) I_{3}^{2}-\frac{1450}{27027} \frac{1}{n^{4}} I_{2}^{3},
\end{gathered}
$$

where $\phi_{0, t w o}$ is the grand potential of the isotropic phase, and we keep terms up to fifth order. Of course, we recover the expansion for the annealed case with $n=T / T_{\lambda}=1$. The Landau multicritical point is still located at 
$\beta_{L}=10 / 3$ and $\mu_{L}=0$, but the sign of the coefficient of $I_{3}^{2}$ depends on the parameter $n$. Indeed, there will be a stable biaxial nematic phase for

$$
n<\frac{108}{143} \approx \frac{2}{3}
$$

which indicates that a slight departure from complete annealing $(n=1)$ is already enough to give rise to a stable biaxial structure. Comparisons with previous results for a Maier-Saupe model with restricted orientations, $n<0.9$, show that a somewhat larger difference between the temperatures is needed to stabilize the biaxial phase in the presence of additional direction fluctuations.

\section{Conclusions}

We have carried out exact statistical-mechanics calculations for a MaierSaupe lattice model with the inclusion of extra disorder degrees of freedom to mimic a mixture of discs and cylinders. The closed-form solutions can be written as a Landau-de Gennes expansion for the free energy in the neighborhood of the transition, with explicit forms of model-dependent coefficients, which allows the use of several results from the literature. The stability of a biaxial nematic structure depends on the treatment of the disorder degrees of freedom. For quenched disorder, with a typical double-delta distribution of discs and cylinder, we obtain a global phase diagram, in terms of temperature and concentration, with a Landau multicritical point, a biaxial and two uniaxial nematic phases. If the disorder degrees of freedom are allowed to reach thermal equilibrium, we show that the biaxial structure becomes unstable. We then assume that orientation and disorder degrees of freedom are coupled to different heat reservoirs, with two different temperatures. In the two-temperature context, we show that a small temperature difference, which is equivalent to a small departure from thermalization, is already enough to stabilize a biaxial nematic structure. These results explain some disagreements in the earlier literature. Also, they qualitatively agree with a previous calculation for a similar Maier-Saupe model with restricted directional orientations.

\section{Acknowledgments}


We thank Eduardo do Carmo and Danilo Liarte for helpful suggestions and comments. This work has been supported by grants from FAPESP and CNPq.

\section{References}

[1] P. G. de Gennes and J. Prost, The Physics of Liquid Crystals, Clarendon Press, Oxford (1993).

[2] E. F. Gramsbergen, L. Longa, and W. H. de Jeu, Phys. Repts. 135, 195 (1986).

[3] S. Chandrasekhar, Liquid Crystals, Cambridge University Press, Cambridge (1992).

[4] S. Singh, Phys. Repts. 324, 107 (2000).

[5] A. M. Figueiredo-Neto and S. R. A. Salinas, The Physics of Lyotropic Liquid Crystals: Phase Transitions and Structural Properties, Oxford University Press, 2005.

[6] M. J. Freiser, Phys. Rev. Lett. 24, 1041 (1970).

[7] R. Alben, J. Chem. Phys. 59, 4299 (1973); Z-Y. Chen and J. M. Deutch, J. Chem. Phys. 80, 2151 (1984).

[8] G. R. Luckhurst, Nature 430, 413 (2004); G. R. Luckhurst, Thin Solid Films 393, 40 (2001).

[9] L. A. Madsen, T. G. Dingemans, M. Nakata, and E. T. Samulski, Phys. Rev. Lett. 92, 145505 (2004); B. R. Acharya, A. Primak, and S. Kumar, Phys. Rev. Lett 92, 145506 (2004); Y. Jang, V. P. Panov, A. Kocot, J. K. Vij, A. Lehmann, and C. Tschierske, Appl. Phys. Lett. 95, 183304 (2009).

[10] L. J. Yu and A. Saupe, Phys. Rev. Lett. 45, 1000 (1980).

[11] Y. Galerne and J. P. Marcerou, Phys. Rev. Lett. 51, 2109 (1983); A. M. Figueiredo-Neto, A. M. Levelut, L. Liebert, and Y. Galerne, Mol. Cryst. Liq. Cryst. 129, 191 (1985); Y. Galerne, A. M. Figueiredo-Neto, 
and L. Liebert, J. Chem. Phys. 87, 1851 (1987); P. A. Santoro, A. R. Sampaio, H. L. F. da Luz, and A. J. Palangana, Phys. Lett. A353, 512 (2006); D. A. Oliveira, D. D. Luders, G. P. Souza, N. M. Kimura, and A. J. Palangana, Cryst. Res. Technol. 44, 1255 (2009); L. Q. Amaral, Liquid Crystals 37, 627 (2010).

[12] P. Palffy-Muhoray, J. R. de Bruyn, and D. A. Dunmur, J. Chem. Phys. 82, 5294 (1985); S. R. Sharma, P. Palffy-Muhoray, B. Bergersen, and D. A. Dunmur, Phys. Rev. A32, 3752 (1985).

[13] E. F. Henriques and V. B. Henriques, J. Chem. Phys. 107, 8036 (1997); E. F. Henriques, C. B. Passos, V. B. Henriques, and L. Q. Amaral, Liquid Crystals 35, 555 (2008).

[14] Y. Rabin, W. E. McMullen, and W. M. Gelbart, Mol. Cryst. Liq. Cryst. 89, 67 (1982); A. Stroobants and H. N. W. Lekkerkerker, J. Phys. Chem. 88, 3669 (1984); F. M. van der Kooij and H. N. W. Lekkerkerker, Phys. Rev. Lett 84, 781 (2000); A. G. Vanakaras, A. F.Terzis, and D. J. Photinos, Mol. Cryst. Liq. Cryst. A362, 67 (2001).

[15] R. Berardi, L. Muccioli, S. Orlandi, M. Ricci, and C. Zannoni, J. Phys.: Condens. Matter 20, 463101 (2008).

[16] E. do Carmo, D. B. Liarte, and S. R. Salinas, Phys. Rev. E81, 062701 (2010).

[17] A. E. Allahverdyan and Th. Nieuwenhuizen, Phys. Rev. E62, 845 (2000); O. M. Ritter, P. C. T. Dajello, and W. Figueiredo, Phys. Rev. E69, 016119 (2004); A. E. Allahverdyan and K. G. Petrosyan, Phys. Rev. Lett. 96, 065701 (2006).

[18] E. do Carmo, A. P. Vieira, and S. R. Salinas, Phys. Rev. E83, 011701 (2011).

[19] D. Allender and L. Longa, Phys. Rev. E78, 011704 (2008).

[20] P. K. Mukherjee and K. Sen, J. Chem. Phys. 130, 141101 (2009).

[21] K. Binder and A. P. Young, Rev. Mod. Phys. 58, 801 (1986); S. K. Ma, Modern Theory of Critical Phenomena, W. A. Benjamin (1976). 